\title{
Antibacterial and antifungal activities of 2,3-pyrrolidinedione derivatives against oral pathogens
}

Atul A. Dhavan ${ }^{\mathrm{a} \S}$, Andrei C. Ionescu ${ }^{\mathrm{b}}$, Rahul D. Kaduskar ${ }^{\mathrm{a}}$, Eugenio Brambilla ${ }^{\mathrm{b}}$, Sabrina Dallavalle $^{\mathrm{a}^{*}}$, Elena Maria Varoni ${ }^{\mathrm{c}}$, Marcello Iriti ${ }^{\mathrm{d}}$

${ }^{a}$ Department of Food, Environmental and Nutritional Sciences, Division of Chemistry and Molecular Biology University of Milan, via Celoria 2, 20133 Milan, Italy.

${ }^{b}$ Department of Biomedical, Surgical and Dental Sciences, IRCCS Galeazzi Hospital, University of Milan, Milan, Italy.

${ }^{c}$ Department of Biomedical, Surgical and Dental Sciences, University of Milan, Milan, Italy.

${ }^{d}$ Department of Agricultural and Environmental Sciences, University of Milan, Milan, Italy.

${ }^{\S}$ Co-first authors

${ }^{*}$ Corresponding author. Tel. +39-02-50316818; Fax: +39-02-50316801

E-mail address: sabrina.dallavalle@unimi.it

\begin{abstract}
Among the novel approaches applied to antimicrobial drug development, natural product-inspired synthesis plays a major role, by providing biologically validated starting points.

Tetramic acids, a class of natural products containing a 2,4-pyrrolidinedione ring system, have attracted considerable attention for their antibacterial, antiviral, antifungal and anticancer activities. On the contrary, compounds with a 2,3-pyrrolidinedione skeleton have been considerably less investigated. In this work, we established chemical routes to the substituted 2,3-pyrrolidinedione core, which enabled the introduction of a wide range of diversity. In the perspective of a potential application for oral healthcare, a number of analogues with various substituents on the 2,3pyrrolidinedione core were investigated for their antimicrobial and antifungal activities. The most promising compound showed a significant antimicrobial activity on Streptococcus mutans and Candida albicans, comparable to that of chlorhexidine, the gold standard in oral healthcare.
\end{abstract}

Keywords: 2,3-pyrrolidinedione, natural product-inspired synthesis, chlorhexidine, Streptococcus mutans, Candida albicans, oral health, antibiofilm activity. 
Oral diseases impose a huge economic burden on society ${ }^{1}$ and dental caries and oral candidiasis are two of the most common, biofilm-related diseases of the oral cavity worldwide.

Dental caries represents a global health problem still far from being completely eradicated. ${ }^{2}$ In this disease, the predominance of Streptococcus mutans- a facultative anaerobic, Gram-positive, acidogenic bacterium- interplays with further caries risk factors, such as frequent sugar intake and hyposalivation, leading to demineralization of hard dental tissues. ${ }^{3}$ In the case of oral candidiasis, Candida albicans, an opportunistic yeast of the oral cavity, proliferates locally producing severe inflammatory lesions of the mucosa, mainly in locally or systemically immunocompromised patients. ${ }^{4}$ The yeast presence has also shown to increase $S$. mutans pathogenicity. ${ }^{5}$ Oral candidiasis in form of prosthetic stomatitis occurs frequently on the palatal mucosa of elderly patients wearing removable prosthesis, due to a local proliferation of the microorganism. Similarly, pseudomembranous oral candidiasis is among those oral lesions strongly associated with HIV infection. ${ }^{2}$ In the case of dental caries, the gold standard acute therapy consists of carious lesion removal and tooth restoration using dental materials, while for oral candidiasis, appropriate antimycotic therapy is recommended.

A preventive approach via antiseptic agents for the control of dental biofilm formation and composition, has recently been proposed to avert both diseases. ${ }^{6,7}$ Chlorhexidine (CHX), a cationic biguanide, is the gold standard for controlling oral biofilm and for oral healthcare, representing the most investigated and prescribed product, commercially available in different forms. However, its use for caries prevention is still largely controversial ${ }^{6,8,9}$, despite its well-reported intense antibacterial action and its ability to firmly adsorb to the tooth structure (pellicle formation on dental hard tissues) and the gingiva (substantivity). ${ }^{10-12}$ Indeed, $\mathrm{CHX}$ is used in vitro as reference biocidal molecule to assess the efficacy of alternative antiseptic agents, showing excellent antibacterial activity against a plethora of oral pathogens, including $S$. mutans $^{13}$, as well as broadspectrum antifungal activity against Candida spp. $^{4}$

Nonetheless, CHX is not exempt from drawbacks, which include both local side effects (such as dental pigmentation and disgeusia) and potential systemic hypersensitivity reactions. ${ }^{8}$ In addition, a reduced level of susceptibility to $\mathrm{CHX}$ by pathogens cannot be a priori excluded, considering also the increasing use of this agent for the oral and hand hygiene. ${ }^{14}$ All together, these issues strongly encourage scientific research in finding alternative antiseptic compounds.

The discovery of new antibacterial and antifungal agents with novel mechanisms of action represents an effective strategy to overcome the limitations related to existing drugs. Interestingly, these agents may also be incorporated inside dental materials. ${ }^{15,16}$ Among the novel approaches applied to antimicrobial drug development, natural product-inspired synthesis plays a major role, by 
providing biologically validated starting points. In this perspective, the re-examination of function and availability of natural products allowed the identification of favoured structures, suitable for drug optimization. ${ }^{17}$

A class of natural products that has attracted considerable attention is that of tetramic acids, containing a 2,4-pyrrolidinedione ring system (compound 1, Figure 1). A tetramic acid is an attractive skeleton because a combination of substituents at different positions can give a large variety of structurally diverse molecules. ${ }^{18}$ Some natural tetramic acid derivatives show remarkable biological activities, ranging from antibacterial and antiviral to antifungal and anticancer ones. Examples include reutericyclin ${ }^{19}$, sintokamide $\mathrm{A}^{20}$, actacystin $^{21}$, streptolydigins ${ }^{22}$, oxazolomycin ${ }^{23}$, janolusimide. $^{24}$

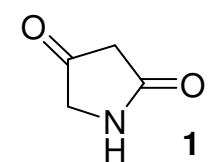

Pyrrolidine-2,4-dione (tetramic acid)

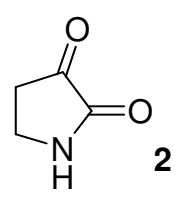

Pyrrolidine-2,3-dione

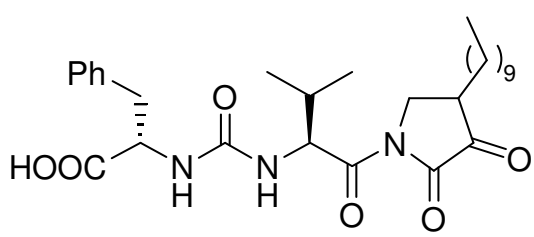

Leopolic Acid A

Figure 1. General structure of 2,3-, 2,4-pyrrolidinediones and Leopolic acid A

Compared to the numerous studies on bioactivities of tetramic acids, compounds with a 2,3pyrrolidinedione skeleton (compound 2, Figure 1) have been considerably less investigated.

To the best of our knowledge, only one natural product (Leopolic acid A, Figure 1) (25 $^{25}$ and a few synthetic compounds ${ }^{26-28}$ have been reported in the literature so far. Therefore, this skeleton can be considered a very attractive target for biological evaluation, offering the possibility of preparing products with several points of diversity, similarly to what occurred for tetramic acid. In this work, we show the results of our preliminary exploration directed towards establishing chemical routes to the substituted 2,3-pyrrolidinedione core, which enabled the introduction of a wide range of diversity. In the perspective of a potential application for oral healthcare, we also assessed the antimicrobial activity of representative compounds on $S$. mutans and C. albicans, and preliminary structure-activity relationships (SAR) have emerged.

We envisaged that the most straightforward route to the synthesis of the 2,3-pyrrolidinedione system could be the Michael addition of a suitably protected amine to ethyl acrylate, followed by a Dieckmann cyclization with diethyl oxalate. ${ }^{29}$ We opted for the $p$-methoxybenzyl (PMB) protecting group, which could be easily removed by cerium ammonium nitrate (CAN) or 2,3-dichloro-5,6dicyanobenzoquinone (DDQ). 
Thus, ethyl acrylate $\mathbf{3}$ was reacted with $p$-methoxybenzylamine to obtain compound $\mathbf{4}$, which was then treated with diethyl oxalate to give 2,3-pyrrolidinedione 5. (Scheme 1). NMR observations showed that the compound exists as an enol tautomer (see SI). Indeed, it has been reported ${ }^{30-32}$ that apparently all 4-monosubstituted 2,3-dioxopyrrolidines are highly enolized, regardless of the nature of the substituent in position 4 .

After obtaining compound $\mathbf{5}$, we planned to prepare a series of analogues to obtain a range of diversity around the heterocyclic core. At first, we decided to compare different functionalities at position 4. The enolic $\mathrm{OH}$ was first protected with a tertbutylsilyl (TBS) group, by treatment of $\mathbf{5}$ with TBSCl, to obtain compound 6.

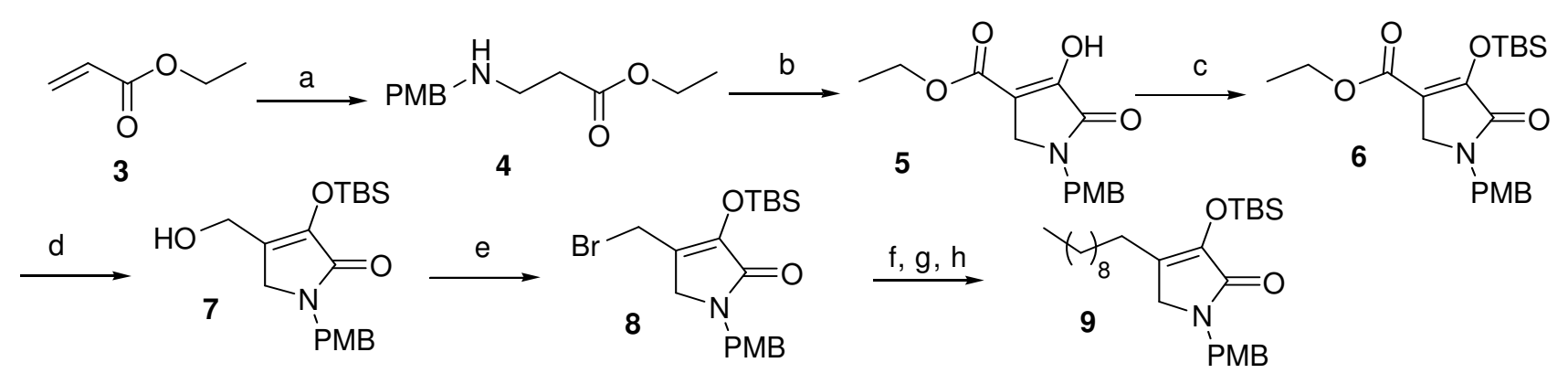

(a) pmethoxybenzylamine, EtOH, rt, 12h, 98\%; (b) diethyloxalate, NaOEt, EtOH, reflux, 3h, 83\%; (c) TBSCl, imidazole, DCM, rt, 6h, 86\%; (d) DIBAL-H, DCM, $-78^{\circ} \mathrm{C}$ to rt, $1 \mathrm{~h}, 72 \%$; (e) $\mathrm{PPh}_{3}, \mathrm{CBr}_{4}, \mathrm{DCM}, \mathrm{rt}, 12 \mathrm{~h}, 87 \%$;

(f) $\mathrm{PPh}_{3}$, toluene, reflux, 6h, 61\%; (g) n-nonanal, LiHMDS, THF, $-78^{\circ} \mathrm{C}$ to rt, 5h, 88\%; (h) $\mathrm{H}_{2} / \mathrm{Pd} / \mathrm{C}, \mathrm{EtOAc}, \mathrm{rt}$, $1 \mathrm{~h}, 89 \%$

Scheme 1. Synthesis of compounds 5-9.

Reduction of $\mathbf{6}$ with diisobutylaluminium hydride (DIBALH) successfully gave alcohol 7 in $72 \%$ yield. Inspired by the 2,3-pyrrolidinedione-containing natural compound Leopolic acid $\mathrm{A}^{25}$ we planned the synthesis of a 4-alkyl substituted derivative. Thus, alcohol 7 was converted into the corresponding bromide by Appel reaction with $\mathrm{PPh}_{3}$ and $\mathrm{CBr}_{4}$. The bromide was reacted with $\mathrm{PPh}_{3}$ to give a bromonium salt which was then subjected to Wittig reaction with a nine-carbons aldehyde. Reduction of the double bond by catalytic hydrogenation gave compound 9 in $89 \%$ yield.

Unfortunately, attempts to remove the PMB group from compounds 6-9 (CAN in ACN /water or DDQ, $\mathrm{CH}_{2} \mathrm{Cl}_{2}$ ) resulted into unstable compounds which decomposed during purification.

Assuming that this instability could be due to the enol protecting group, we decided to use a benzyl group as an alternative. Thus, compound $\mathbf{5}$ was treated with benzyl bromide to obtain compound $\mathbf{1 0}$ (Scheme 2). To compare the benzyl group-containing compounds to analogues with the TBS group on the enolic $\mathrm{OH}$, we repeated the same sequence reported above (see Scheme 1) using compound 
10 instead of compound 6 (Scheme 2). Reduction with DIBALH, followed by Appel bromination and Wittig reaction, allowed the introduction in position 4 of the aliphatic chain (compound 13).

Deprotection of PMB group from compound $\mathbf{1 0}$ was successful and gave 14. Having the compound with a free $\mathrm{NH}$ in hands, we explored the effect of a polar group linked to nitrogen, to be compared with compound 10, carrying a lipophylic PMB group. Thus, 14 was acylated with activated Nprotected valine, to obtain 15. Removal of the Boc protecting group afforded 16, containing the free aminoacid residue.

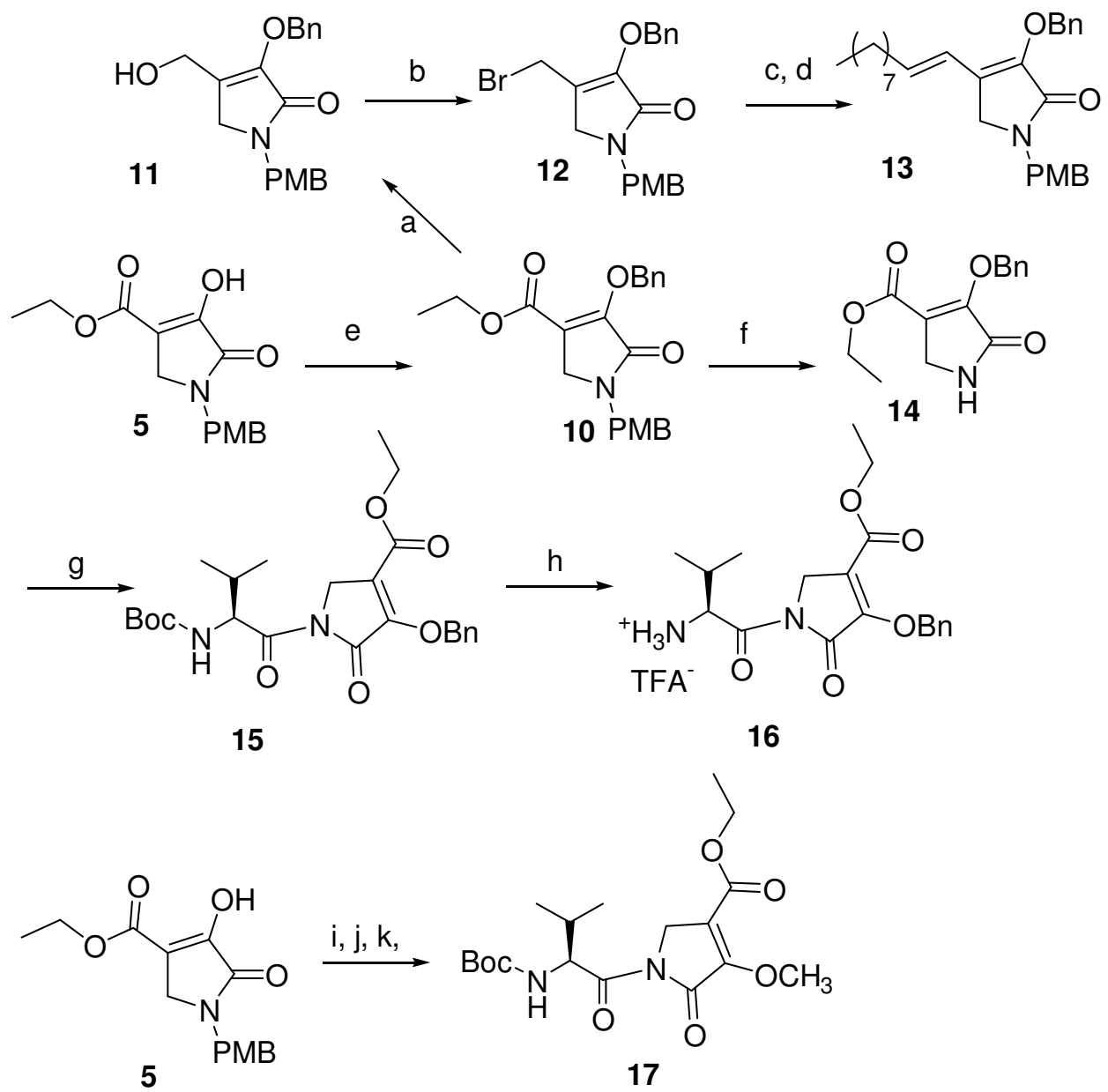

(a) DIBAL-H, DCM, $-78^{\circ} \mathrm{C}$ to rt, $1 \mathrm{~h}, 56 \%$; (b) $\mathrm{PPh}_{3}, \mathrm{CBr}_{4}, \mathrm{DCM}, \mathrm{rt}, 3 \mathrm{~h}, 52 \%$; (c) $\mathrm{PPh}_{3}$, toluenene, reflux, 6h, 75\%; (d) n-nonanal, LiHMDS, THF, $-78^{\circ} \mathrm{C}$, $5 \mathrm{~h}, 52 \%$; (e) $\mathrm{BnBr}, \mathrm{K}_{2} \mathrm{CO}_{3}$, $\mathrm{DMF}, \quad \mathrm{rt}, \quad \mathrm{h}, \quad 50 \%$; (f) $\mathrm{CAN}, \mathrm{ACN}$ : water, rt, $1 \mathrm{~h}, 73 \%$; $(\mathrm{g})$ 2-tert-butoxycarbonylamino-3-methyl-butyric acid pentafluorophenyl ester, BuLi, THF, $-78^{\circ} \mathrm{C}$ to rt, $0.5 \mathrm{~h}, 86 \%$; (h) TFA, DCM, $0^{\circ} \mathrm{C}, 0.5 \mathrm{~h}, 90 \%$; (i) $\mathrm{K}_{2} \mathrm{CO}_{3}$, DMF, $0{ }^{\circ} \mathrm{C}, 15 \mathrm{~min}$, then Mel, rt, 1h, 61\%; (j) $\left(\mathrm{NH}_{4}\right)_{2} \mathrm{Ce}\left(\mathrm{NO}_{3}\right)_{6}, \mathrm{ACN}$ : $\mathrm{H} 203: 1,0{ }^{\circ} \mathrm{C}$ then rt, $1 \mathrm{~h}, 71 \%$; (k) BuLi, THF, $-78{ }^{\circ} \mathrm{C}$, $10 \mathrm{~min}$, then (S)-Tert-butyl- 1-((pentafluorophenoxy)carbonyl)-2-methylpropylcarbamate, -78 ${ }^{\circ} \mathrm{C}, 30 \mathrm{~min}, 80 \%$.

Scheme 2. Synthesis of compounds 10-17. 
The prepared compounds were investigated for their antimicrobial and antifungal activities. The results are shown in Figures 2 (antibacterial activity against $24 \mathrm{~h}$ S. mutans biofilm) and 3 (antifungal activity against $24 \mathrm{~h}$ C. albicans biofilm) and in Table S1 (see SI).

A $0.2 \%$ solution of compound $\mathbf{5}$, containing a free enolic $\mathrm{OH}$, gave a $51.3 \%$ inhibition of $S$. mutans growth. On C. albicans biofilm, however, the inihibition rate did not reach 50\%, even at the highest concentration tested (1\%). The protection of the enolic OH by a TBS group (6) decreased the antimicrobial activity of the compound, whereas a benzyl group in the same position (10) totally deprived the compound of any antibacterial activity.

Reduction of the ester group to introduce a polar alcohol moiety at position 4 of both TBS and benzyl substituted compounds yielded derivatives 7 (showing a mild antibacterial and antifungal activity) and $\mathbf{1 1}$ (deprived of antibacterial activity, it showed a mild antifungal activity). Compound $\mathbf{1 1}$ showed indeed a similar activity to the corresponding ester 10, whereas compound $\mathbf{7}$ showed a decreased activity compared to the ester 6.

Having not gained potency by introducing a polar group, we replaced the ester group with an apolar aliphatic chain to obtain 9 and 13. Comparing the activities of 6 to 9 , we observed that the introduction of the aliphatic chain deprived the derivative of antibacterial activity, but almost doubled its antifungal effect. Interestingly, the introduction of the apolar chain on the benzyl substituted derivative $\mathbf{1 3}$ resulted in the highest antibacterial activity recorded, about $93 \%$ of growth inhibition against $S$. mutans biofilm, not significantly different from CHX when diluted up to $0.2 \%$ w/v ( $p=1.0000)$. Derivative 13 also showed the highest antifungal activity, significantly higher than that of CHX $(\mathrm{p}<0.0001)$ when tested in its highest concentration $(1 \%)$.

After that, the effect of substituents on the nitrogen atom was investigated. Removal of the PMB group from compound $\mathbf{1 0}$ to obtain compound $\mathbf{1 4}$ did not significantly change its antibacterial and antifungal activities. An unexpected gain in antibacterial activity was observed with the introduction of the protected aminoacidic moiety to obtain compound $\mathbf{1 5}$. This compound had only slightly lower, although non significantly different, antibacterial and antifungal activity when compared to compound $\mathbf{1 3}$, and, when in its highest concentration, had similar activity to CHX on the tested biofilms.

Removal of the Boc protecting group to yield compound $\mathbf{1 6}$ resulted in a significant decrease in antibiofilm activity.

Finally, considering the substantial activity showed by compound 15, we synthesized the corresponding derivative with a methyl in place of the benzyl group to yield compound 17. This replacement, however, caused another drop in activity, suggesting that the presence of a lipophylic benzyl group in position 3 was productive in terms of antibiofilm activity against the tested strains. 
When comparing the antibacterial and antifungal activity of the different compounds, it is interesting to note that, as opposed to the effect on $S$. mutans biofilms, most compounds showed fungicidal activity and, in particular, derivatives 10, 11, 13, 14, 15 and 17 exhibited a concentrationdependent activity on $C$. albicans biofilm. The TBS-substituted compounds $(\mathbf{6}, 7,9)$ had lower antifungal activity than the corresponding benzyl-substituted compounds, which, in turn, showed an overall comparable antimicrobial and antifungal activity.

In conclusion, we have developed a chemical strategy for the synthesis of substituted 2,3pyrrolidinedione-containing compounds, which enabled the introduction of a wide range of diversity. The antimicrobial activity of representative compounds on $S$. mutans and C. albicans has been assessed and preliminary structure-activity relationships (SAR) have emerged.

The results obtained suggest that compounds possessing a 2,3-pyrrolidinone skeleton can be considered promising candidates in the development of new antibacterial and antifungal compounds. In particular, compound 13 showed a significant antibiofilm activity against both tested strains. This activity was comparable to that of $\mathrm{CHX}$, the gold standard in dental healthcare. Further biological tests and SAR studies on this new series of compounds are currently underway, in the perspective of a potential application in oral healthcare. 
Figure 2. Streptococcus mutans viable biomass after $1 \mathrm{~h}$ exposure to different concentrations of the tested derivatives. The negative control (the solution used to dilute the derivatives) is shown as a green bar and green dashed line while the positive control (a $0.2 \%$ solution of Chlorhexidine) is highlighted in red. Data are presented as \% of biofilm-forming, living microbial cells \pm 1 standard error, assuming the negative control as $100 \%$ viability. The threshold of at least $50 \%$ of inactivation of microbial cells is also shown as orange dashed line.

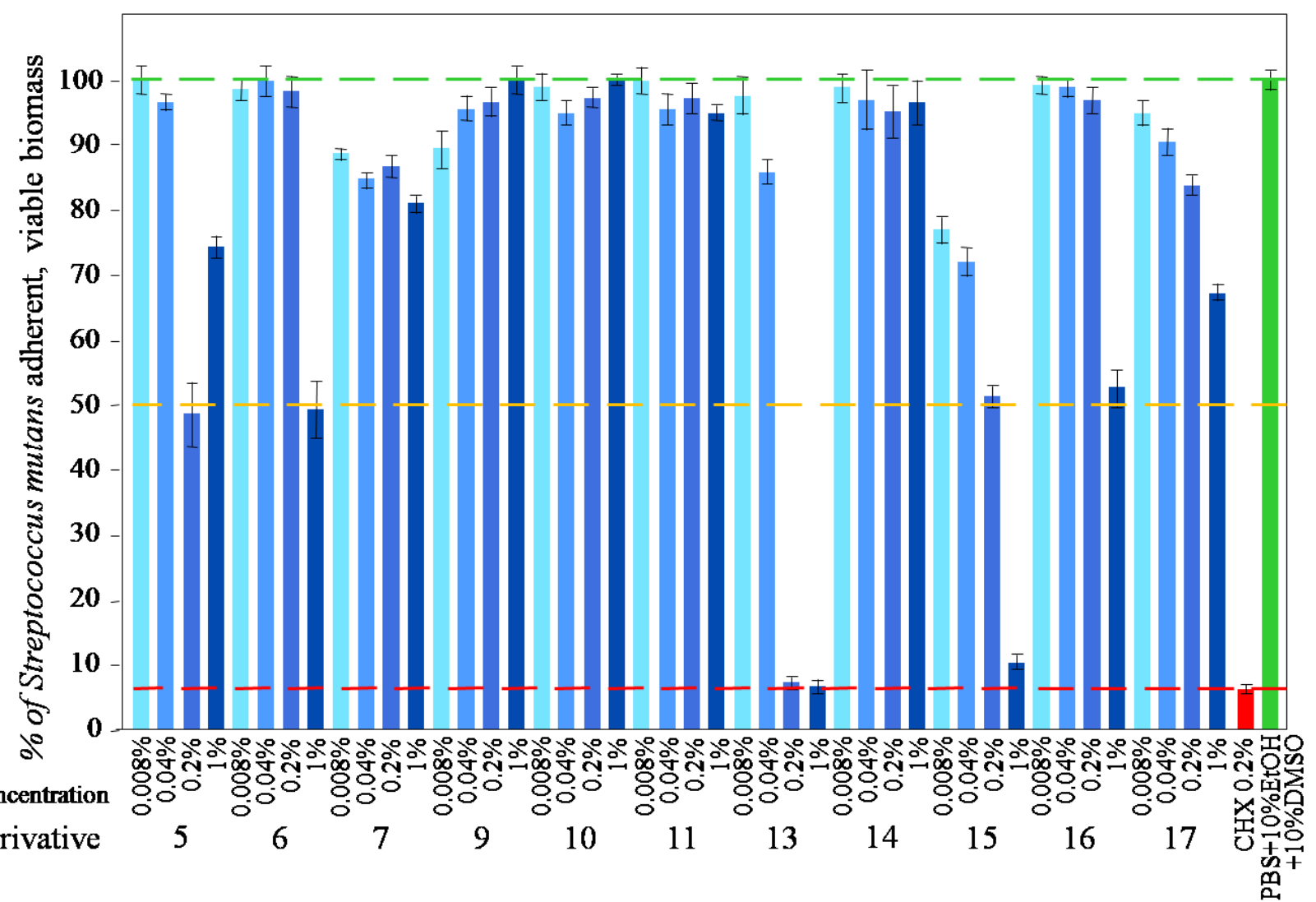


Figure 3. Residual Candida albicans viable biomass after $1 \mathrm{~h}$ exposure to different concentrations of the tested derivatives. The negative control (the solution used to dilute the derivatives) is shown as a green bar and green dashed line while the positive control (a $0.2 \%$ solution of Chlorhexidine) is highlighted in red. Data are presented as $\%$ of biofilm-forming, living microbial cells \pm 1 standard error, assuming the negative control as $100 \%$ viability. The threshold of at least $50 \%$ of inactivation of microbial cells is also shown as orange dashed line.

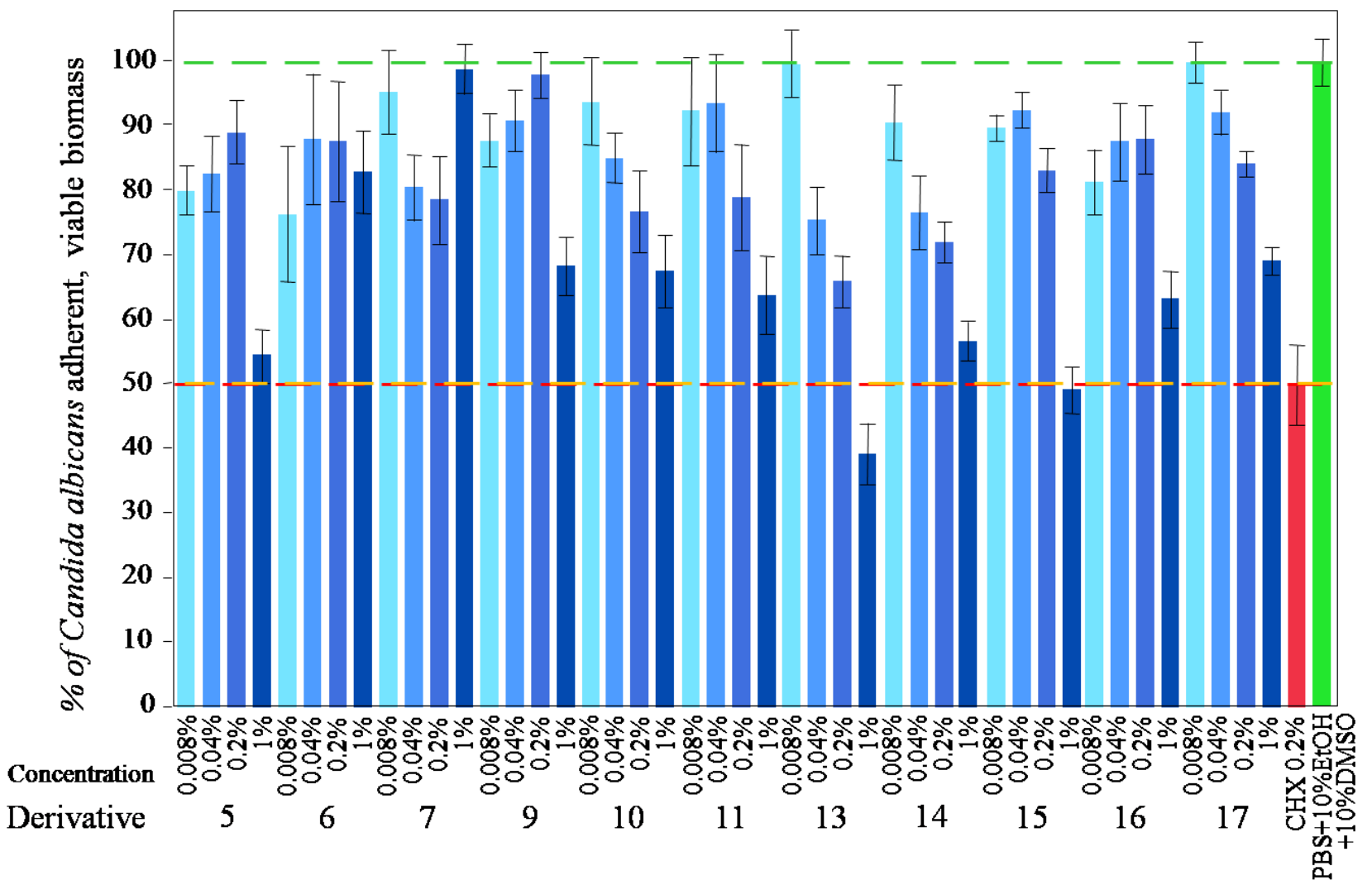

\section{Supplementary data}

Supplementary data associated with this article can be found, in the online version, at

\section{References and notes}

1 Listl, S.; Galloway, J.; Mossey, P. A.; Marcenes, W. J. Dent. Res. 2015, 94, 1355-61.

2. Petersen P.E.; Bourgeois, D.; Ogawa, H.; Estupinan-Day S.; Ndiaye, C. Bull. World Health Organ. 2005, 83, 661.

3. Metwalli, K.H.; Khan, S.A.; Krom, B.P.; Jabra-Rizk, M.A. PLoS Pathog. 2013, 9, e1003616.

4. Salim, N.; Moore, C.; Silikas, N.; Satterthwaite, J.; Rautemaa, R. Int. J. Antimicrob. Agents 2013, 41,65 .

5. Falsetta, M. L.; Klein, M. I.; Colonne, P. M.; Scott-Anne, K.; Gregoire, S.; Pai, C. H.; Koo, H. Infection and Immunity 2014, 82, 1968. 
6. Walsh, T.; Oliveira-Neto, J. M.; Moore, D. In Cochrane Database of Systematic Reviews. 2015, 4, CD008457

7. Budtz-Jörgensen, E. Acta Odontol. Scand. 1990, 48, 61.

8. Varoni, E.; Tarce, M.; Lodi, G.; Carrassi, A. Minerva Stomatol. 2012, 61, 399.

9. Autio-Gold, J. Oper. Dent. 2008, 33, 710.

10. Auschill, T. M.; Hein, N.; Hellwig, E.; Follo, M.; Sculean, A.; Arweiler, N. B. J. Clin. Periodontol. 2005, 32, 147.

11.Van Strydonck, D. A.; Timmerman, M. F.; van der Velden, U.; van der Weijden, G.A. J. Clin. Periodonto . 2005, 32, 305.

12. Adams, D.; Addy, M. Adv. Dent. Res. 1994, 8, 291.

13. Emilson, C. G. J. Dent. Res. 1994, 73, 682.

14. Horner, C.; Mawer, D.; Wilcox, M. J. Antimicrob. Chemother. 2012, 67, 2547.

15. Brambilla, E.; Ionescu, A.; Mazzoni, A.; Cadenaro, M.; Gagliani, M.; Ferraroni, M.; Tay, F.; Pashley, D.; Breschi, L. Dent. Mater. 2014, 30, 926.

16. Brambilla, E.; Ionescu, A.; Fadini, L.; Mazzoni, A.; Imazato, S.; Pashley, D.; Breschi, L.; Gagliani, M. J. Adhes. Dent. 2013, 15, 431.

17. Brown, D. G.; Lister, T.; May-Dracka, T. L. Bioorg. Med. Chem. Lett. 2014, 24, 413.

18. Jeong, Y. C.; Anwar, M.; Bikadi, Z.; Hazai, E.; Moloney, M. G. Chem. Sci. 2013, 4, 1008.

19. Ganzle, M. G. Appl. Microbiol. Biotechnol. 2004, 64, 326.

20. Sadar, M. D.; Williams, D. E.; Mawji, N. R.; Patrick, B. O.; Wikanta, T.; Chasanah, E.; Irianto, H. E.; Soest, R. V.; Andersen, R. J. Org. Lett. 2008, 10, 4947.

21. Pattenden, G.; Rescourio, G. Org. Biomol. Chem. 2008, 6, 3428.

22. Pronin, S. V.; Martinez, A.; Kuznedelov, K.; Severinov, K.; Shuman, H. A.; Kozmin, S. A. J. Am. Chem. Soc. 2011, 133, 12172.

23. Bagwell, C. L.; Moloney, M. G.; Thompson, A. L. Bioorg. Med. Chem. Lett. 2008, 18, 4081.

24. Giordano, A.; Monica, C. D.; Landi, F.; Spinella, A.; Sodano, G. Tetrahedron Lett. 2000, 41, 3979.

25. Raju, R.; Gromyko, O.; Fedorenko, V.;Luzhetskyy, A. ; Müller, R. Tetrahedron Lett, 2012, 53, 6300.

26. Southwick, P. L.; Crouch, R. T. J. Am. Chem. Soc. 1953, 76, 3413.

27. Reddy, T. R. K.; Li, C.; Guo, X.; Myrvang, H. K.; Fischer, P. M.; Dekker, L. V. J. Med. Chem. 2011, 54, 2080.

28. Mylari, B. L.; Beyer, T. A.; Siegel, T. W. J. Med. Chem. 1991, 34, 1011. 
29. Coumar, M. S.; Wu, J. S.; Leou, J. S.; Tan, U. K.; Chang, C. Y.; Chang, T. Y.; Lin, W. H.; Hsu, J. T. A.; Chao, Y.S .; Wu, S. Y.; Hsieh, H. P. Bioorg. Med. Chem. Lett. 2008, 18, 1623.

30. Meyer, W. L.; Vaughan, W. R. J. Org. Chem. 1957, 22, 1554.

31. Vaughan, W. R.; Covey, I. S. J. Am. Chem. Soc. 1958, 80, 2197.

32. Southwick, P. L.; Barnas, E. F. J. Org. Chem. 1962, 27, 98. 